\title{
Investigation of Heat Transfer and Fluid Mechanics across a Heated Rotating Circular Cylinder in Crossflow
}

\author{
G. F. Smaisim ${ }^{1,2}$, O. M.H. Fatla ${ }^{1,3}$, A. Valera-Medina1, A.M. Rageb ${ }^{4}$, N. Syred ${ }^{1 *}$ \\ ${ }^{1}$ School of Engineering, Cardiff University, Cardiff, United Kingdom \\ ${ }^{2}$ Mechanical Engineering Dept., Kufa University, Iraq \\ ${ }^{3}$ Mechanical Engineering Dept., Al-Qadisya University, Iraq \\ ${ }^{4}$ Mechanical Engineering Dept., Basrah University, Iraq \\ *Corresponding author email: syredn@cardiff.ac.uk
}

\begin{abstract}
Considerable attention has been given to flows passing over bluff bodies such as cylinders. This is due to their importance in applications such as heat exchanger, cooling towers and electronic circuit cooling. It has been proved that flow performance and heat transfer could be improved remarkably if the cylinders rotate and flow stabilizers/blockers are used to increase residence time and Nusselt number. This is due to the effect of the vortical structures in the wake region on the drag and heat transfer coefficients. Therefore, forced convection heat transfer across stationary and rotating horizontal cylinder dissipating uniform heat flux was investigated numerically and experimentally in this work. The maximum values of rotational and crossflow Reynolds numbers attained were 960 and 160 , respectively. The non-dimensional rotational velocity $(\alpha)$ was varied from 0 to 6 . Finite volume numerical simulations using ANSYS were performed. Average temperatures of the cylinder surface were measured by using a thermal camera and the flow profile was captured by High Speed Photography and LDV, offering new experimental results for correlation purposes. Results show that the maximum average heat transfer rate occurs at the same Reynolds number whilst the minimum value occurs at the higher rotational Reynolds number. At higher rotational Reynolds numbers the Nusselt number is nearly independent of Reynolds number and thermal boundary conditions. Comparison with previous numerical studies showed good agreement.
\end{abstract}

Keywords: Rotating cylinder, crossflow, heat transfer, experimental visualization, CFD

\section{Introduction}

Flow across a stationary circular cylinder has drawn the attention of researchers in fluid dynamics for decades. Cylinders are considered ideal samples of bluff bodies that can provide a better understanding of vortex shedding phenomena and vortex interaction in downstream flows. Numerous studies of flows passing stationary and rotating cylinders in quiescent mediums have been investigated by several authors [1-6]. However, these studies have been only analytical approaches. Although this technique plays an important role in flow control, boundary layer control, reduction of heat transfer and lift enhancement, limited works have been done to correlate these analytical analyses. These fundamental could be eventually expended to include cooling of gas turbine rotors or electrical motor shafts, rotating condensers for seawater distillation, cooling of high-speed gas bearings, space vehicle technology, spinning projectiles, nuclear reactors, heat exchangers, drying of paper on rollers in the paper industry, electronic packaging, power electronic devices, rotating machineries, viscous pumps, rotating electrodes, etc. Thus, good understanding of the phenomena related to these flows could have considerable practical applications.

For flow passing a rotating cylinder, the results not only depend on Reynolds number, $R e=U_{\infty} D / v$; but also depend on the velocity at the cylinder surface normalized by the free-stream velocity, $\alpha=\omega D / 2 U_{\infty}$. Numerous studies have investigated the effect of the cylinder's rotation. Ingham and Tang [7] numerically calculated the drag and lift coefficients of a flow between $\mathrm{Re}=5$ and 20 crossing a rotating cylinder with a rotational rate of $0 \leq \alpha \leq 3$. Stojkovic et al. [8] reported the effect of high rotational rates of a circular cylinder on both lift and drag forces. Their study investigated the steady and unsteady flows at low Re between $0.01 \leq$ 
$R e \leq 45$ considering rotation rates of $0 \leq \alpha \leq 12$. It was proved that the rotation rate had a significant role in suppression of the development of vortex shedding. Costa and Raimundo [9] showed that the cylinder rotation significantly affects heat transfer as a result of thermo-physical properties. The study was carried out numerically using a cylinder centered inside a square enclosure with constant temperature at its vertical walls. Chuan and Chao [10] performed a numerical simulation to determine the influence of stationary and rotating cylinders on natural and mixed convections. The study was conducted on a circular cylinder centered in a square enclosure with aspect ratios $(\mathrm{L} / \mathrm{D})=1.67,2.5$ and 5. Other parameters such as $\operatorname{Pr}=0.07,0.7$ and 7, and $\mathrm{Ra}=10^{4}, 10^{5}$ and $10^{6}$ were also studied. Sachin and Atul [11] numerically examined the changes in flow and heat transfer characteristics with $\mathrm{Re}=20$ to 160 passing over a rotating cylinder of constant wall temperature (CWT). Rotation rates of $\alpha \leq 6$ and an aspect ratio (L/D) $=100$ were considered. Following their previous work in 2009, they examined the effect of new boundary condition of Uniform Heat Flux (UHF) on heat transfer from the cylinder surface [12].

The objective of this work is to provide experimental results, which have not been provided until now, to correlate some of these findings and current CFD studies developed by our group. Flow and heat performance of a stationary and rotating circular cylinder placed in a forced convection laminar flow were studied with a great variety of techniques.

\section{Experimental setup}

In the present work, the governing parameters are: $\mathrm{Re}=80,120$ and 160 , rotational rate $\alpha=0,2,4,6$. Streamline patterns, velocity, temperature distributions and Nusselt number describe the phenomena of vortex shedding under the effect of different rotational velocities and three different Reynolds numbers.

Experiments were performed in a wind tunnel with dimensions and layout as shown in Fig. 1. A horizontal circular cylinder of diameter $\mathrm{D}=12 \mathrm{~mm}$ located inside the tunnel was used. A uniform flow was forced to pass over the cylinder by a suction fan fixed at the narrow end of the test rig. The velocity at the outlet of the rig was measured by hot wire anemometers. The continuity equation was involved in calculation of inlet velocities and Reynolds numbers.

A variable speed DC motor model MT63B14-2 was used to drive the cylinder. The range of rotating speed was between 0 and $3725 \mathrm{rpm}$ in counterclockwise direction. An inverter that adjusts the supplied electric current and frequency was used to control the rotational speed. Heating of the cylinder was accomplished by inserting a cylindrical electrical heater of $10 \mathrm{~mm}$ diameter and $150 \mathrm{~mm}$ length. The heater was connected to the mains power supply through a slip ring. It was adjusted through a variac controller at 40 Volt and $200 \mathrm{~mA}$. In order to prevent heat transfer from the cylinder ends, two disks of an insulated material were used.

Laser Doppler Velocimetry (LDV) was used with a 543-TSI-A01 system and a PDM 1000 model processor to examine 2- D velocity profiles. In all cases smoke was utilized as scattering particles, since the densities of the smoke and air were close enough that allow a good particles suspension. A 450500 model probe was calibrated at $45^{\circ}$ from the horizontal to reduce the dispersion of the laser beams by the bearing and the bearing house. It was mounted on a traverse mechanism. The measuring volume was located at $30 \mathrm{~mm}$ height from the cylinder. Ten measurement points were obtained for all cases spaced at $10 \mathrm{~mm}$ between each other, Fig. 2. The results were analyzed using the FlowSizer software. Flow turbulence was also characterized using the LDV data.

Infrared Thermography was also used. A H2630 camera equipped with 640 x 480 pixels infrared detectors was utilized. The camera was placed at a distance of $1000 \mathrm{~mm}$ from the target and the optical axis was perpendicular to the object of interest at a height of $600 \mathrm{~mm}$ from the ground for all cases. All the images have been processed utilizing the InfRec Analyzer NS9500 Lite software. It is important for the study of local Nu number to measure the temperature distribution in the boundary layer around the cylinder. Therefore, ten K-type thermocouples were installed in different positions above the cylinder, Fig. 3, to measure the temperature of air close to the cylinder.

A Photron Fastcam High Speed Camera model APX RS of 250,000 frames/sec maximum speed velocity was also used. In order to avoid any resolution problem and to increase the visual field, the camera was setup at only 
$1000 \mathrm{frames} / \mathrm{sec}$. The camera was positioned at $1500 \mathrm{~mm}$ from the cylinder. The images were analyzed using the PFV 531 software. Similarly to the LDV, smoke was also used to provide insights of the flow paths.

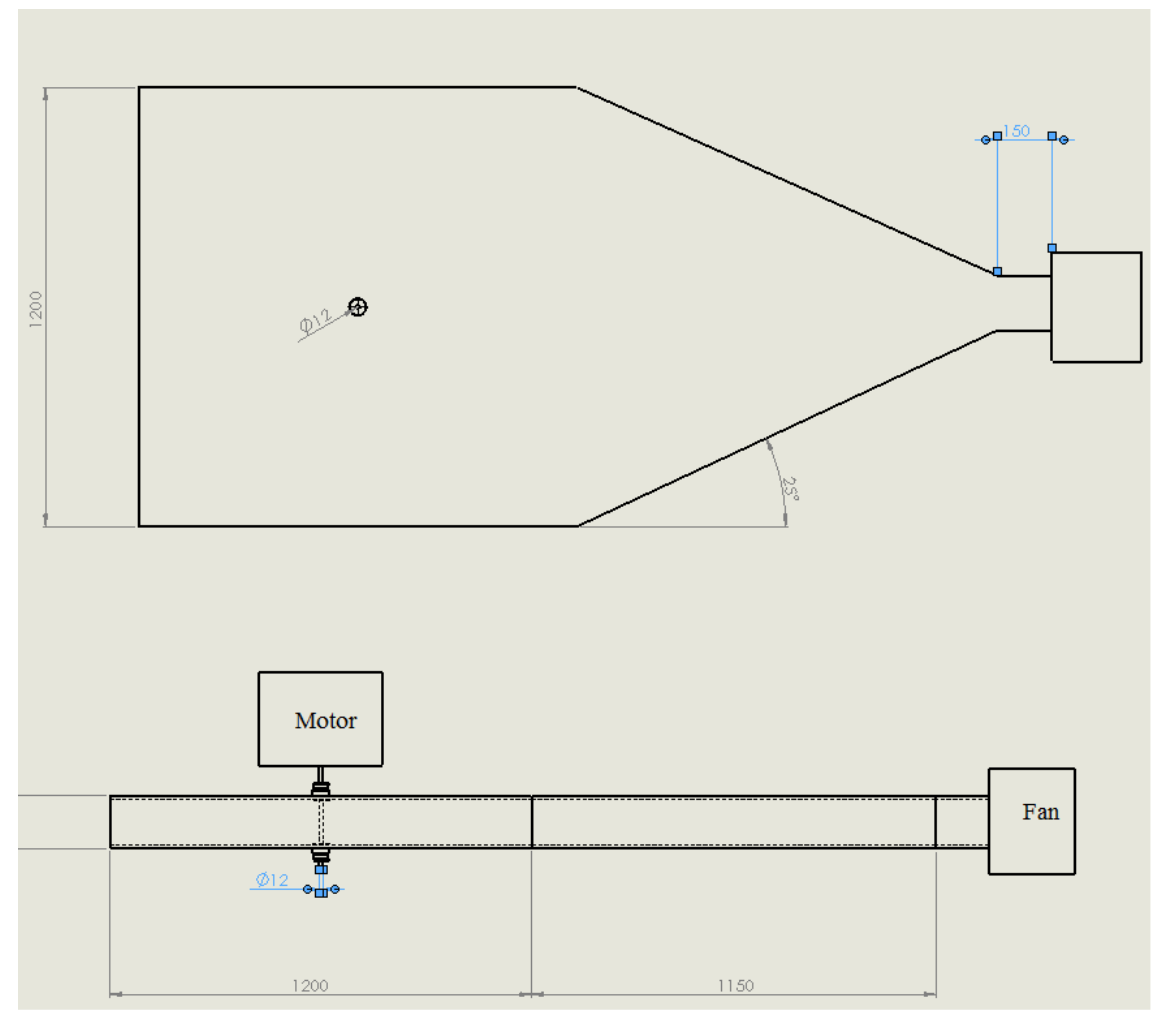

Fig. 1 Test system's layout and dimensions

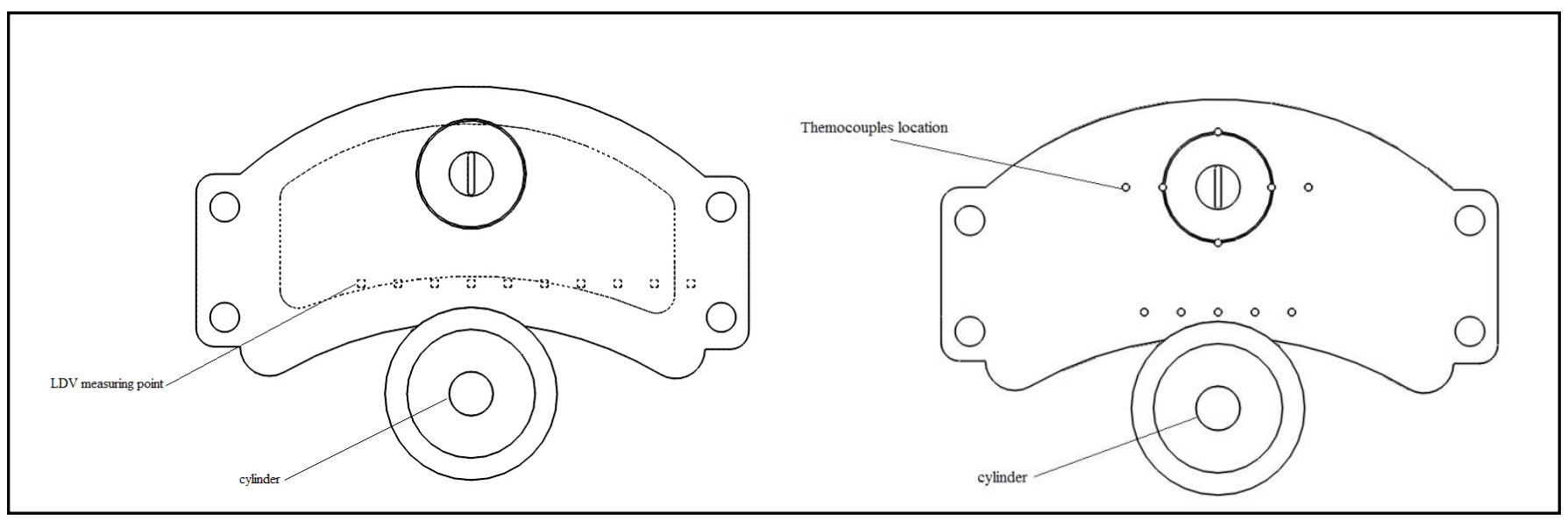

Fig. 2 LDV measurement points.

Fig. 3 Thermocouples locations

\section{Numerical Analysis}

\subsection{Governing equation and boundary conditions}

The governing equations used in this study is based on pre-conditioned three-dimensional, steady and incompressible Navier-Stokes equations, which are expressed in the following form for continuity, momentum and energy: 
$\frac{\partial U}{\partial X}+\frac{\partial V}{\partial Y}+\frac{\partial W}{\partial Z}=0$

$\frac{\partial(U U)}{\partial X}+\frac{\partial(V U)}{\partial Y}+\frac{\partial(W U)}{\partial Z}=-\frac{\partial P}{\partial X}+\frac{1}{R e}\left(\frac{\partial^{2} U}{\partial X^{2}}+\frac{\partial^{2} U}{\partial Y^{2}}+\frac{\partial^{2} U}{\partial Z^{2}}\right)$

$\frac{\partial(U V)}{\partial X}+\frac{\partial(V V)}{\partial Y}+\frac{\partial(W V)}{\partial Z}=-\frac{\partial P}{\partial Y}+\frac{1}{R e}\left(\frac{\partial^{2} V}{\partial X^{2}}+\frac{\partial^{2} V}{\partial Y^{2}}+\frac{\partial^{2} V}{\partial Z^{2}}\right)$

$\frac{\partial(U W)}{\partial X}+\frac{\partial(V W)}{\partial Y}+\frac{\partial(W W)}{\partial Z}=-\frac{\partial P}{\partial X}+\frac{1}{R e}\left(\frac{\partial^{2} W}{\partial X^{2}}+\frac{\partial^{2} W}{\partial Y^{2}}+\frac{\partial^{2} W}{\partial Z^{2}}\right)$

$\frac{\partial(U \theta)}{\partial X}+\frac{\partial(V \theta)}{\partial Y}+\frac{\partial(W \theta)}{\partial Z}=\frac{1}{R e P r}\left(\frac{\partial^{2} \theta}{\partial X^{2}}+\frac{\partial^{2} \theta}{\partial Y^{2}}+\frac{\partial^{2} \theta}{\partial Z^{2}}\right)+q$

Where all velocities and coordinates in the above equations are converted to the dimensionless form by the freestream velocity $\mathrm{u}_{\infty}$ and the cylinder diameter $\mathrm{D}$.

\subsection{Boundary Conditions}

In order to solve the governing equations (1) to (5), boundary conditions need to be set to define the study case. At the solid surface of the cylinder Dirichlet non-slip boundary conditions were applied. The cylinder was kept at constant and uniform heat flux. The flow is assumed to be incompressible with constant thermo-physical properties. At the inlet of the flow, the velocities were adjusted to cover Reynolds numbers of 80, 120 and 160. Slip boundary conditions were implemented for the square enclosure.

\subsection{Numerical method}

In the present work, a three-dimensional laminar version of finite volume method was used to solve the governing equations (1) to (5). Flow simulation was performed using ANSYS Fluent code. A great deal of attention was given to the distributions and numbers of grid points in order to capture all the features of the flow separation and wake formation. A grid structure of a total number of control volumes of 34554 was found to be grid independent and efficient to achieve accurate solutions, convergence and numerical stability. In order to obtain an adequate examination of the flow feature around the cylinder and to improve the accuracy of the numerical results without loss of efficiency, local grid refinement near the cylinder wall was employed. The Simple Implicit Method (SIMPLE) was used in order to achieve mass conservation and to avoid pressure-velocity decoupling. For the purpose of convergence acceleration, a convergence criteria of $10^{-6}$ was used for all residuals.

\section{Results and discussion}

\subsection{CFD streamline patterns}

Fluid flow patterns are shown near the cylinder in Fig. 4 at $\operatorname{Re}=80,120$ and 160 with $\alpha=0-6$. The streamlines for a stationary cylinder $(\alpha=0)$ show a pair of stable vortices (top clockwise and bottom counterclockwise) in the wake region behind the cylinder at $\mathrm{Re}=80$ whose size increase with $\mathrm{Re}$. As the cylinder starts rotating, the flow becomes asymmetric under the influence of cylinder counterclockwise rotation due to the interaction between the flow generated close to the cylinder and the free-stream flow, Fig. 4. It was noted that the stagnation point shifts to the top of the cylinder. As the dimensionless speed of the cylinder increases, for a fixed $\mathrm{Re}$, the region of close streamlines around the cylinder extends further away from the wall. Therefore, the stagnation point moves upwards from the cylinder. The rotation also results in the merging of the counterclockwise vortex with the rotation of the cylinder to form a vortex around the cylinder. The clockwise vortex separates from the back surface of the cylinder to form an enveloping and a detached vortices [12], Fig. 5 at $\alpha=1$.

At $\alpha \geq 3$ under all Re the clockwise-detached vortex disappears by merging into the stream and only the enveloping vortex can be seen within the stream structure. At low rotational speed the flow pattern near the cylinder is strongly affected by viscous forces, whereas at high values of $\alpha$ the flow is dominated by inertial forces. 
Experimentally the vortex shedding activity was visualized using a smoke generator, then captured using High Speed Photography. The smoke rotates under the influence of the cylinder counterclockwise rotation as the flow moves from the left to the right side. The smoke paths for a flow at Reynolds numbers of 80,120 and 160 passing over the cylinder with a rotational speed $\alpha=6$ is shown in Fig. 6.

$\mathrm{Re}=80$
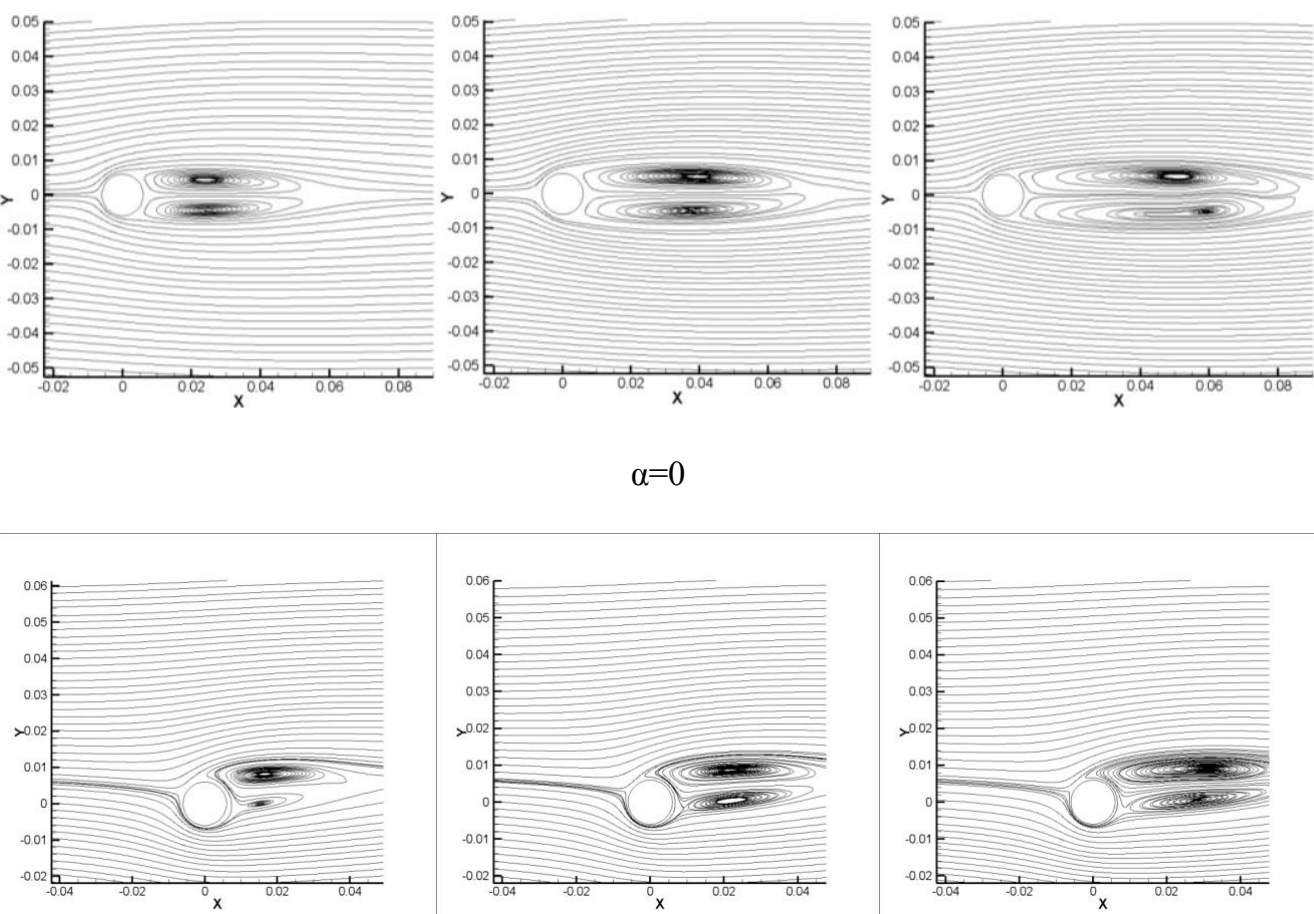

$\alpha=1$
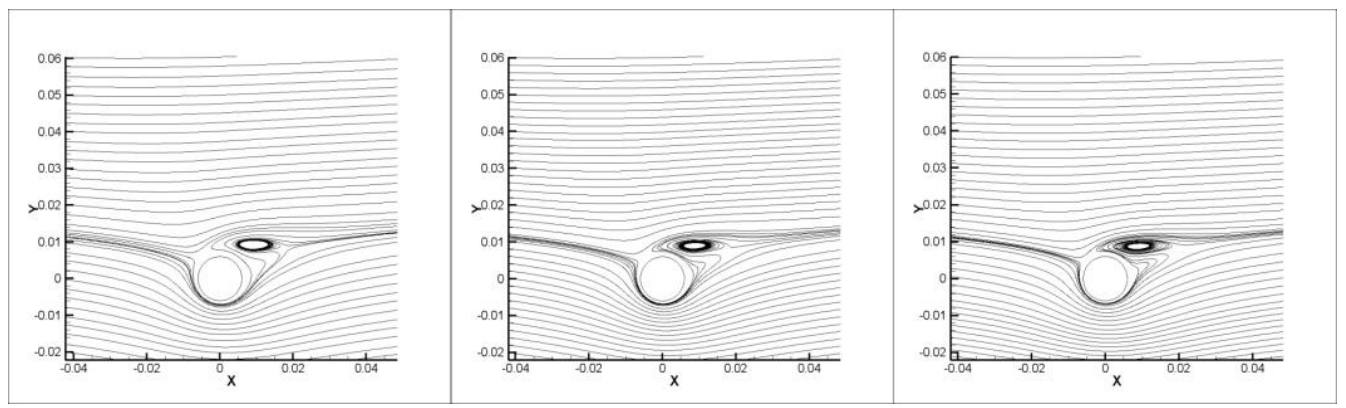

$\alpha=2$

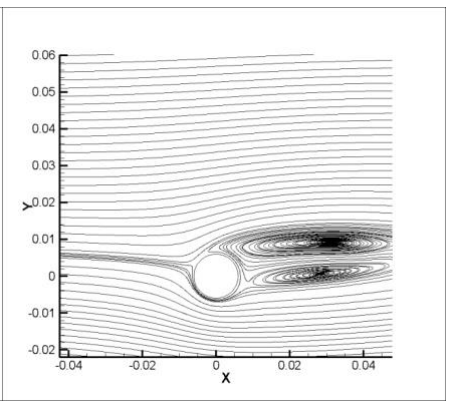



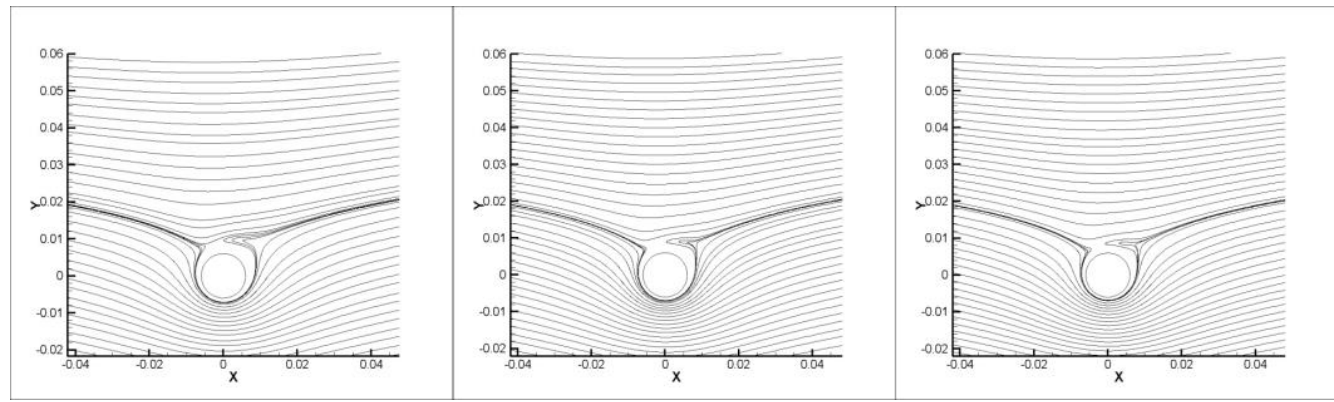

$\alpha=3$
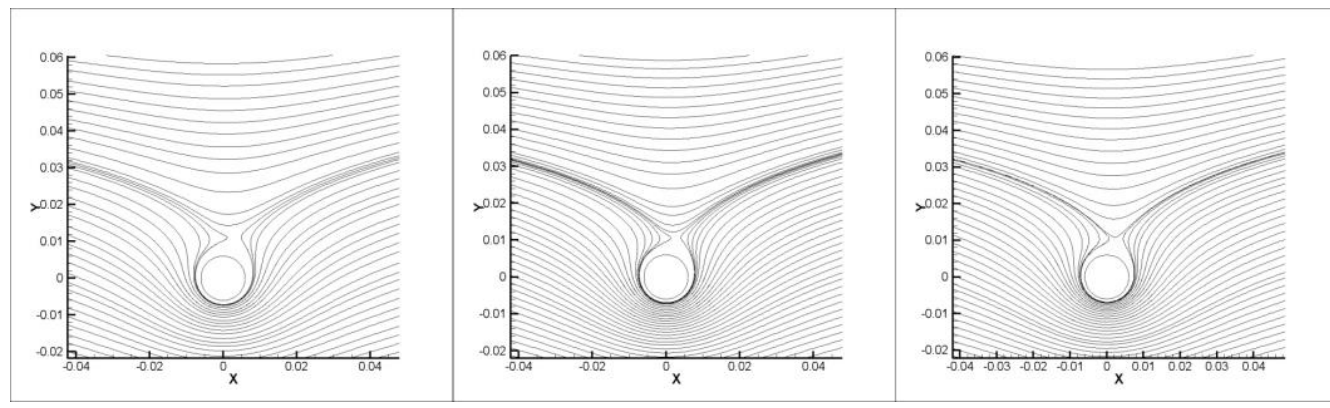

$\alpha=4$
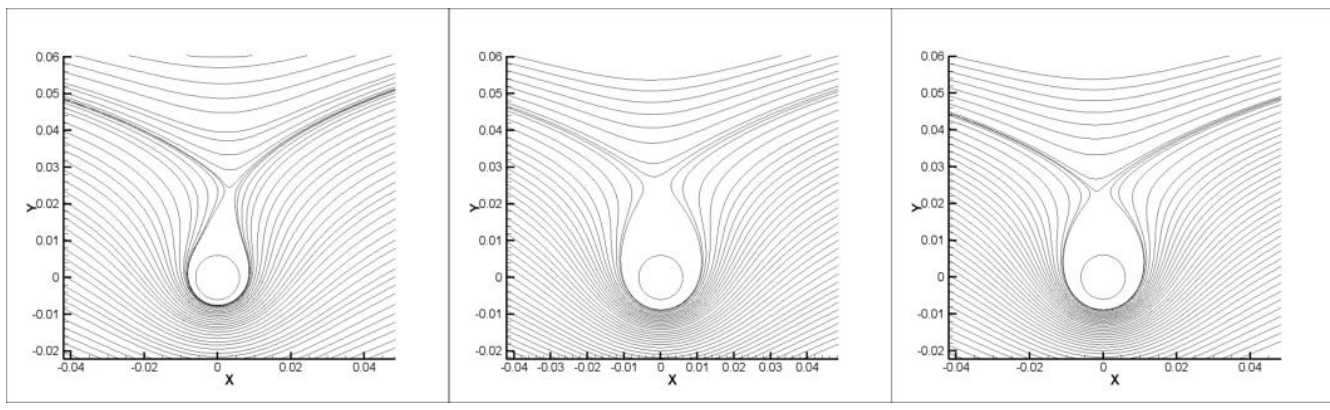

$\alpha=5$
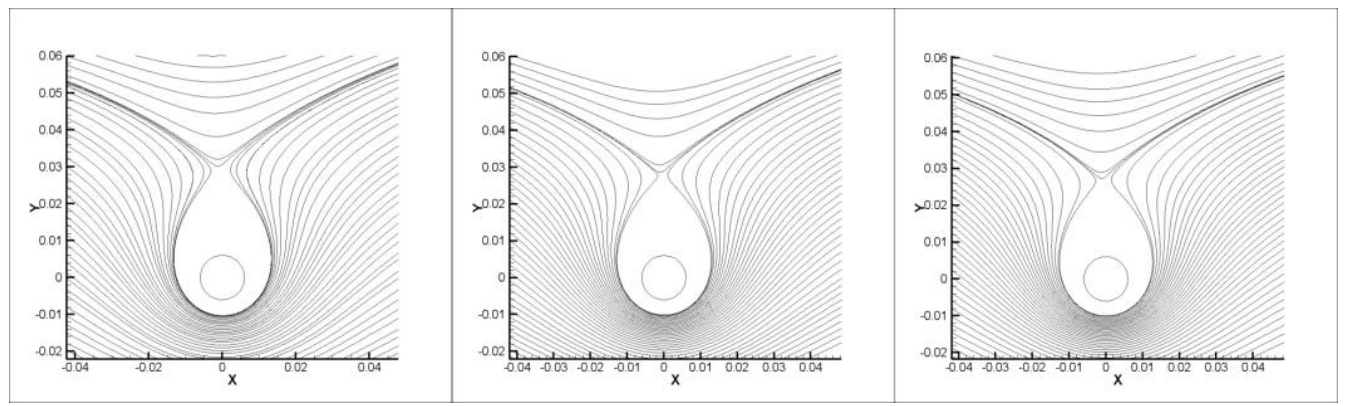

$\alpha=6$

Fig. 4 Streamlines near Cylinder for $\mathrm{Re}=80,120$ and 160, $\alpha$ 0-6. Similar to [12] 

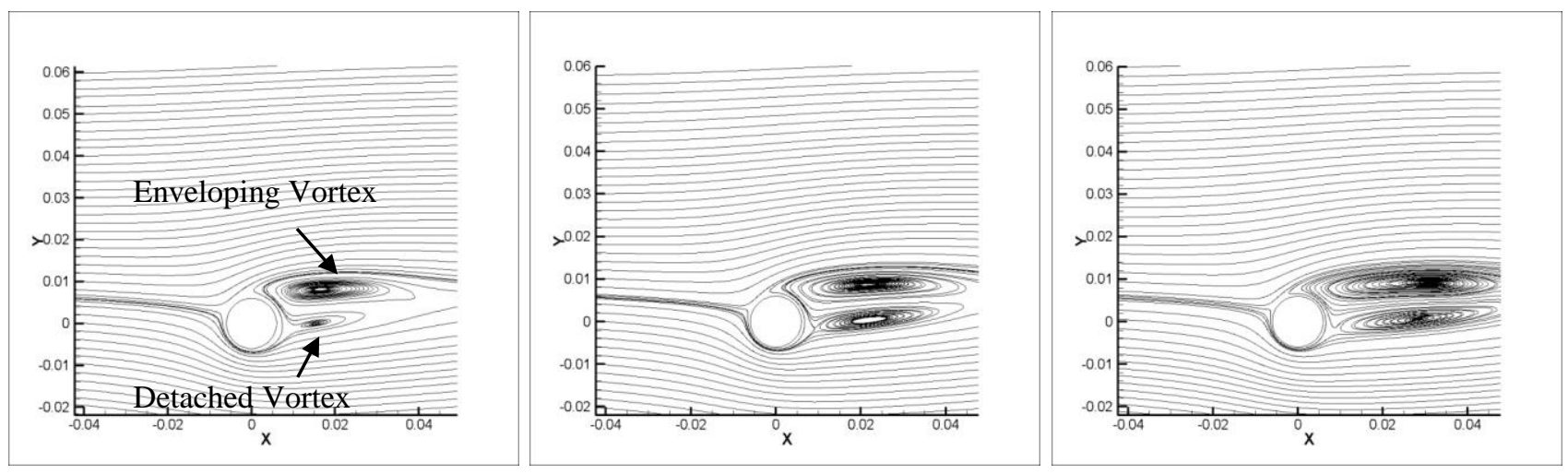

Fig. 5. Enveloping and Detached Vortex at $\alpha=1$ for $R e=80,120$ and 160
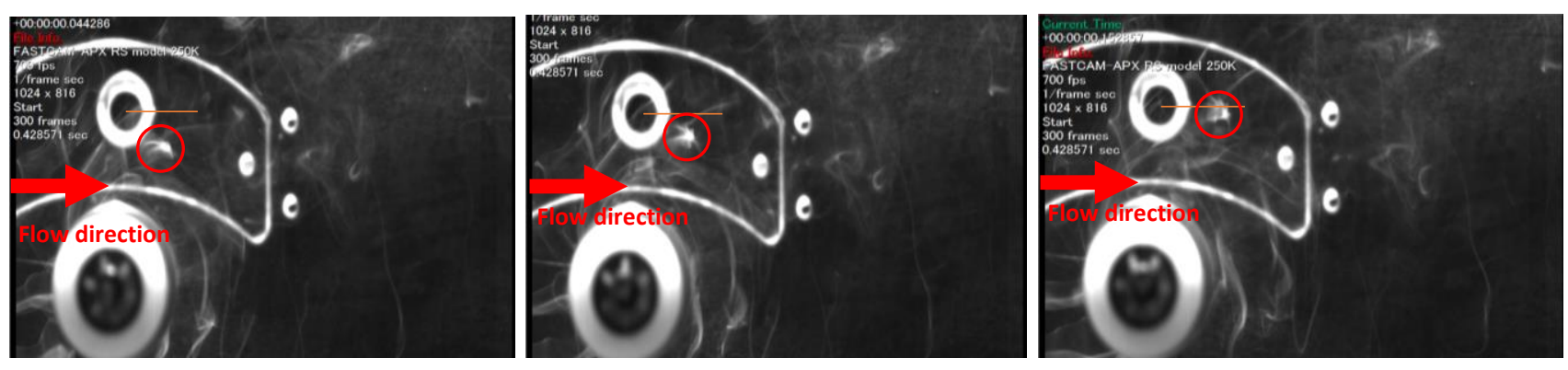

Fig. 6 High Speed Photography at $\mathrm{Re}=80,120$ and 160. If is evident how the stagnation point (encircled) has moved upwards based on Re.

\subsection{Velocity Distribution}

For all cases the first three measurement points were located upstream the cylinder on X coordinates -0.03 , -0.02 and -0.01 . The fourth point was located where the center of the cylinder is positioned. Finally, the other six points $(0.01,0.02,0.03,0.04,0.05$ and 0.06$)$ were located downstream the cylinder.

The two velocity components are plotted separately for different Reynold and rotational rates. At $\alpha>0$, the results show fluctuations in velocity components before the cylinder and relatively steady state conditions after the cylinder. This seems to be caused by the rotation of the cylinder that suppresses the vortex shedding. When the cylinder rotates, a continuous layer of fluid attached to the cylinder surface rotates with the cylinder, consequently moving the stagnation point. This creates a turbulence zone before the cylinder and calm conditions downstream [15-17]. Furthermore, at $\alpha>0$ the Vx velocity decreases, Figs. 7-10, especially in the immediate region upstream the rotating cylinder. This is due to the movement of the stagnation point above the cylinder. The local velocity in the Y-direction also decreases in the region close to the cylinder for the same reason, but starts to increase in the region just behind the cylinder as a result of the rotation of the stream as a consequence of the cylinder counterclockwise rotation, Figs. 7-10. 

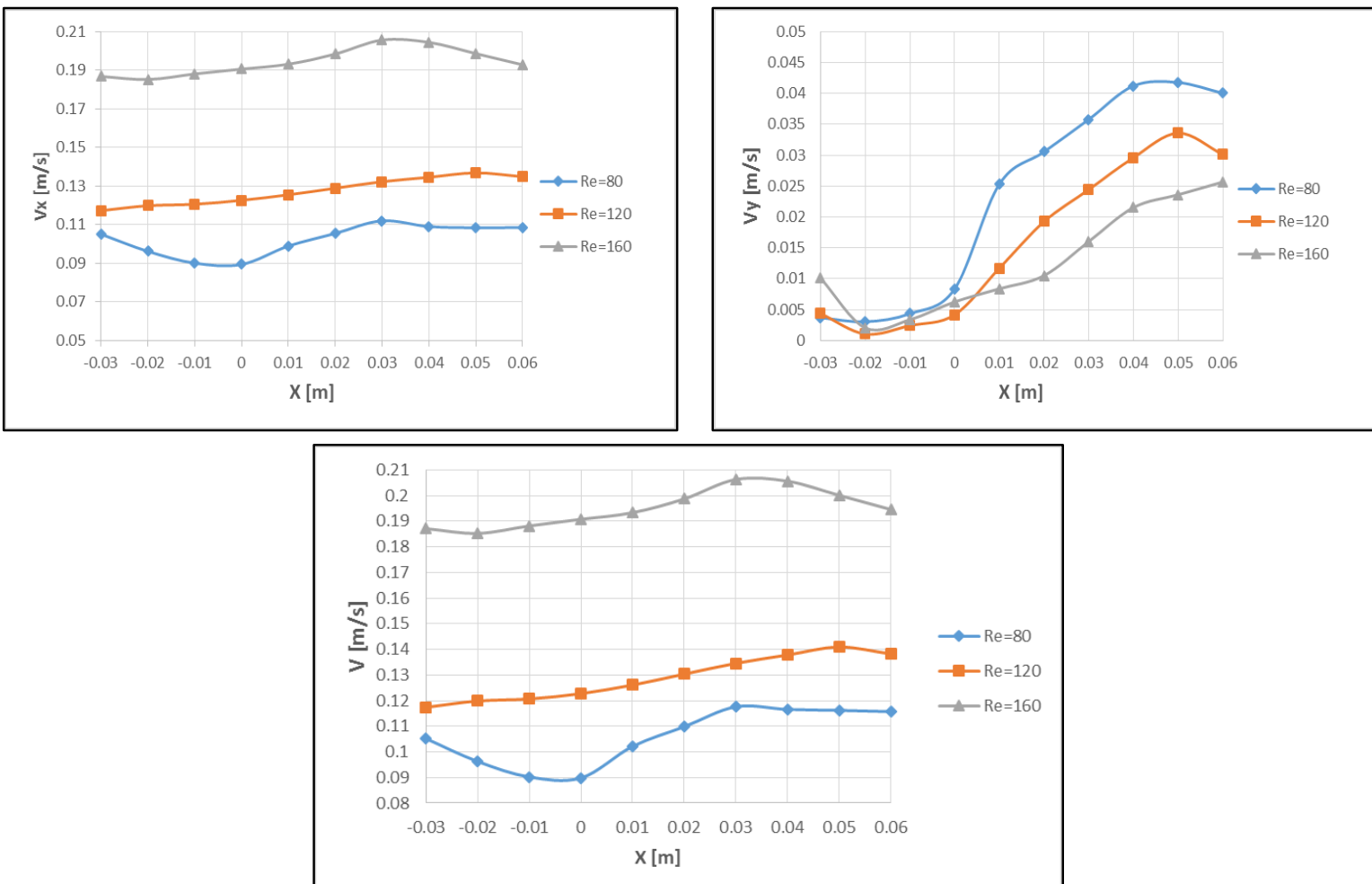

Fig. 7. Velocity Distribution (Vx, Vy and Resultant Velocity) at $\mathrm{Re}=80,120$ and 160 with $\alpha=0$.

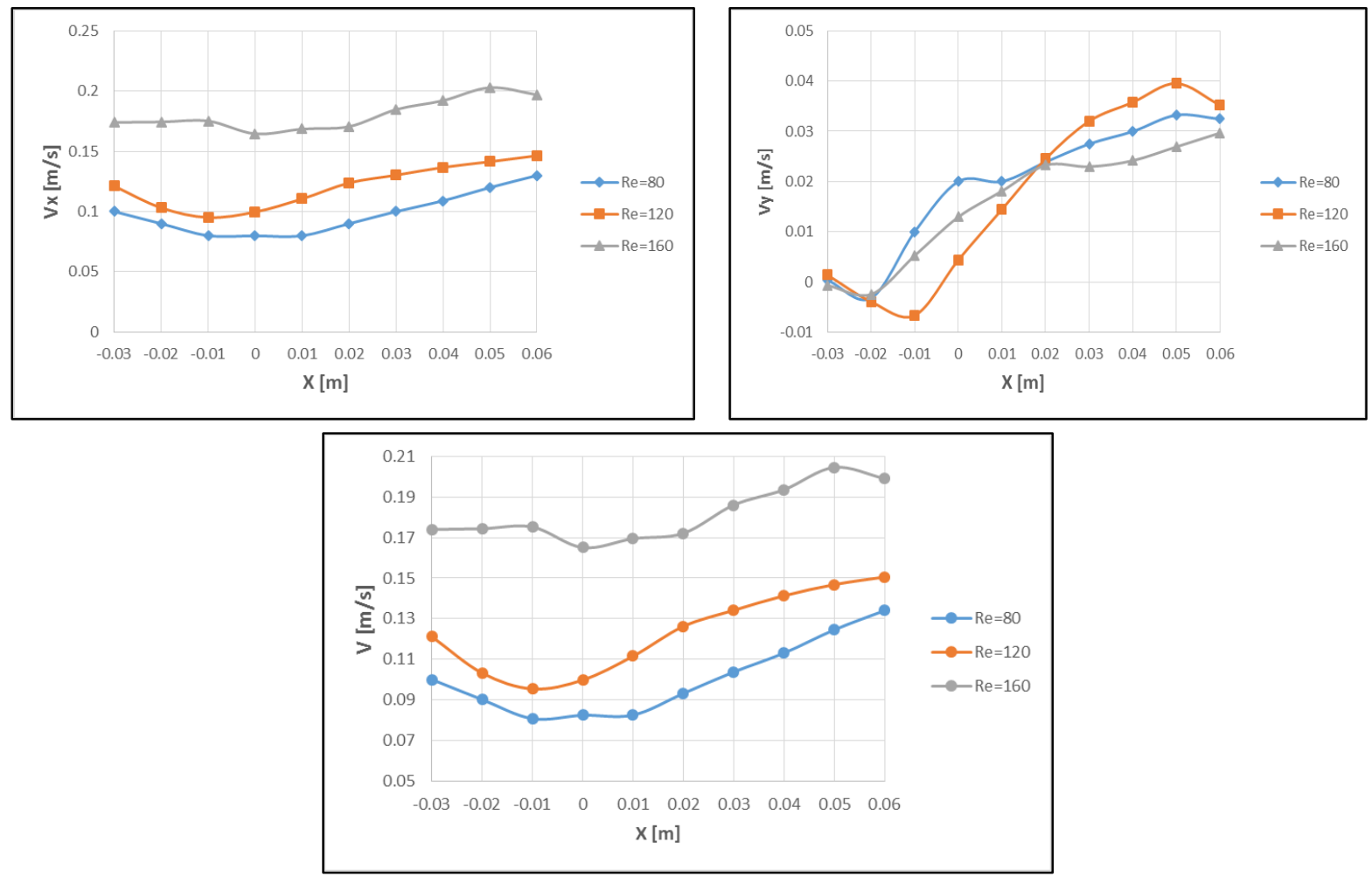

Fig. 8. Velocity Distribution (Vx, Vy and Resultant Velocity) at $\mathrm{Re}=80,120$ and 160 with $\alpha=2$. 

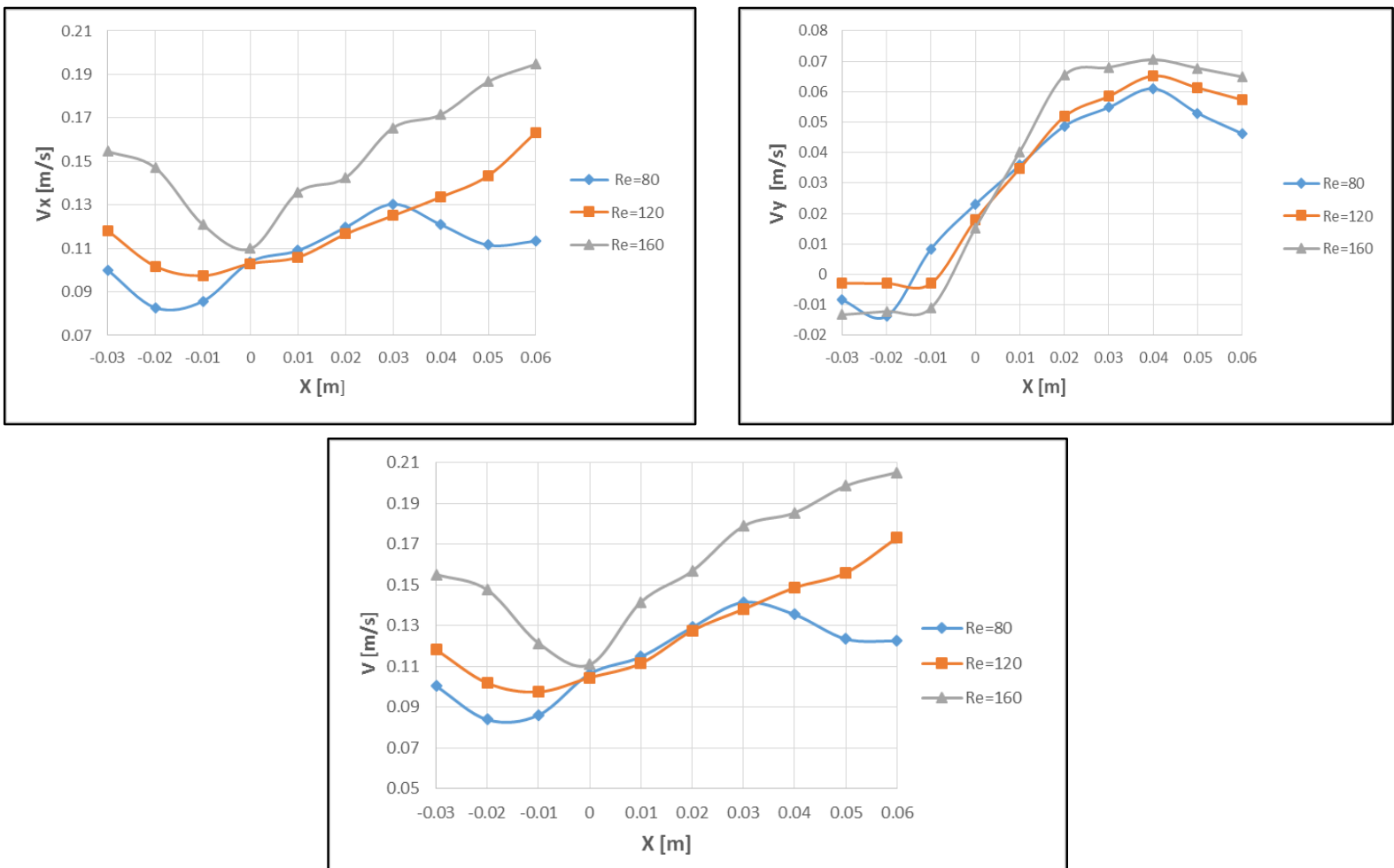

Fig. 8. Velocity Distribution (Vx, Vy and Resultant Velocity) at $\mathrm{Re}=80,120$ and 160 with $\alpha=4$.
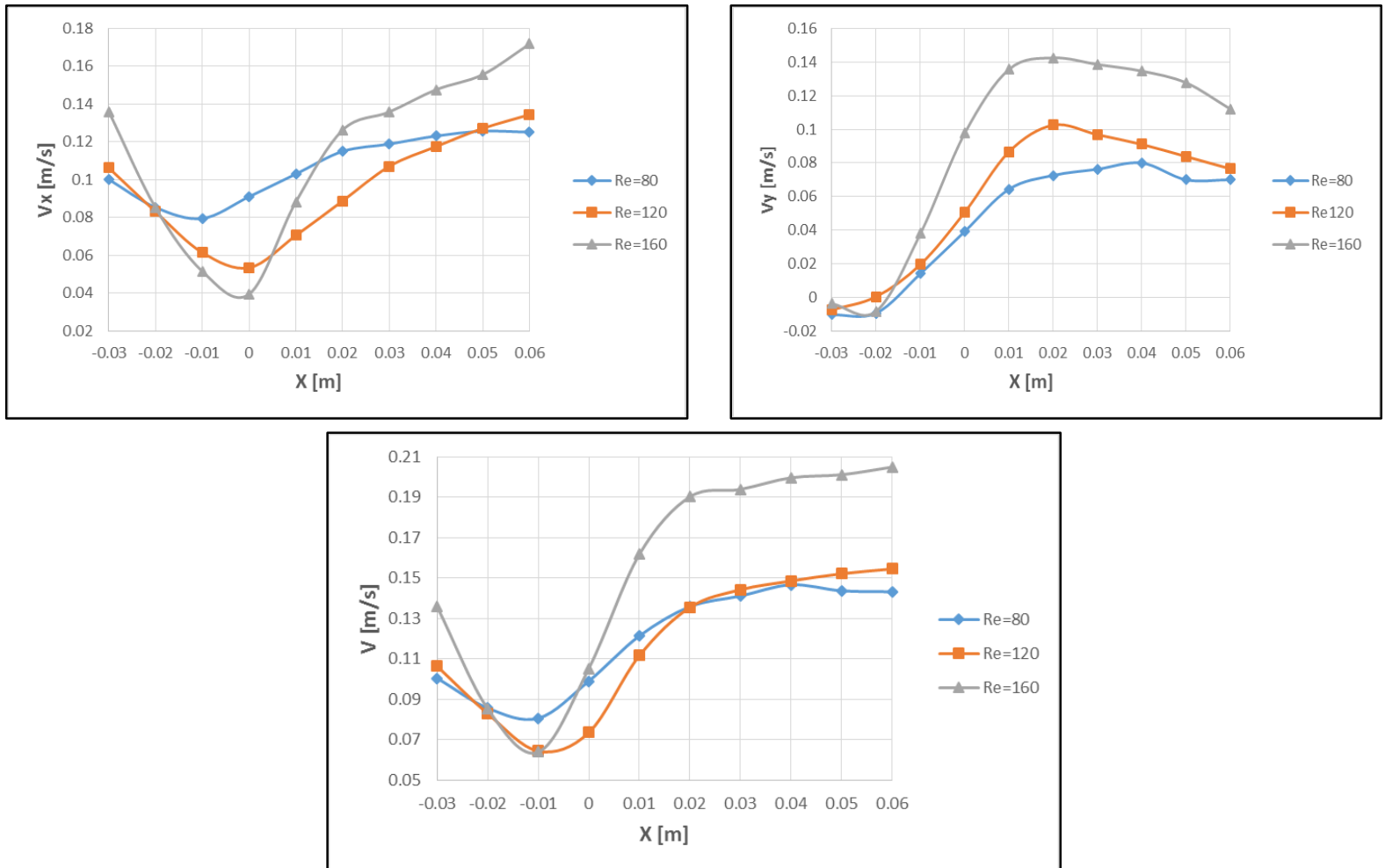

Fig. 9. Velocity Distribution (Vx, Vy and Resultant Velocity) at $\mathrm{Re}=80,120$ and 160 with $\alpha=6$. 


\subsection{Turbulence intensity}

Although the present work deals with low and intermediate Reynolds numbers, it is impossible to avoid turbulent. Turbulence intensity (T.I.) was calculates using the following equation:

$$
T . I .=\frac{u^{\prime}}{U}
$$

Where:

$$
\begin{aligned}
& \mathrm{u}^{\prime}=\text { the Root-Mean-Square (RMS) } \\
& u^{\prime}=\sqrt{\frac{1}{N} \sum_{i=1}^{N}(u i-U)^{2}} \\
& \mathrm{~N}=\text { total number of particles } \\
& \mathrm{u}_{\mathrm{i}}=\text { velocity of particle } \\
& T . I .=\sqrt{(T \cdot I \cdot x)^{2}+\left(T \cdot I_{\cdot}\right)^{2}}
\end{aligned}
$$

Park and Lee [13] calculated the turbulence intensity in their experimental work of flow crossing a finite stationary circular cylinder to detect the vortex formation length. They defined the end of the vortex formation region as the point that has a maximum velocity fluctuations. In the present work the turbulence intensity was calculated to examine the effect of cylinder rotation on vortex formation. The results were obtained using LDV.

Figs. 10 and 11 show values of T.I. under various conditions. It can be noted that some values have exceeded $100 \%$, this is caused by the low average air speed passing over the cylinder and the large fluctuation presented at the relevant location. The results also show that the cylinder rotation reduces the fluctuation behind the cylinder. Due to the rotation of the cylinder the rotating flow intersects the upstream flow developing a zone of high turbulence before the cylinder, whilst the flow remains smoother after the cylinder. The streamlines in Badr et al. [14] and Ingham et al. [7] support the explanation, although their work dealt with lower rotational rates.

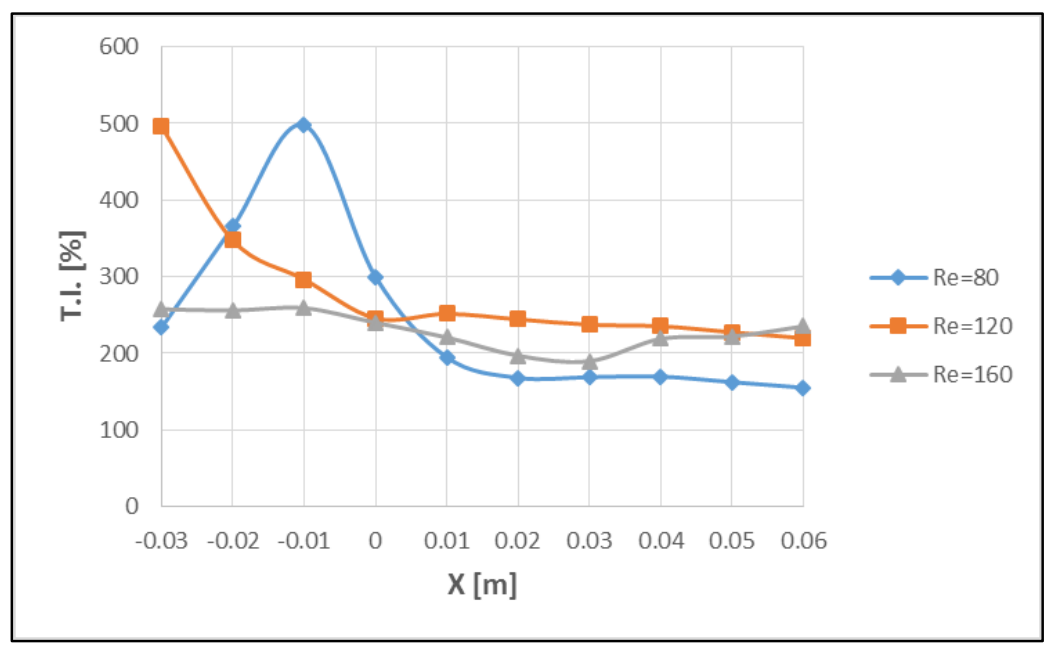

Fig. 10. Turbulence intensity for $\operatorname{Re}=80,120$ and 160 at $\alpha=0$ 


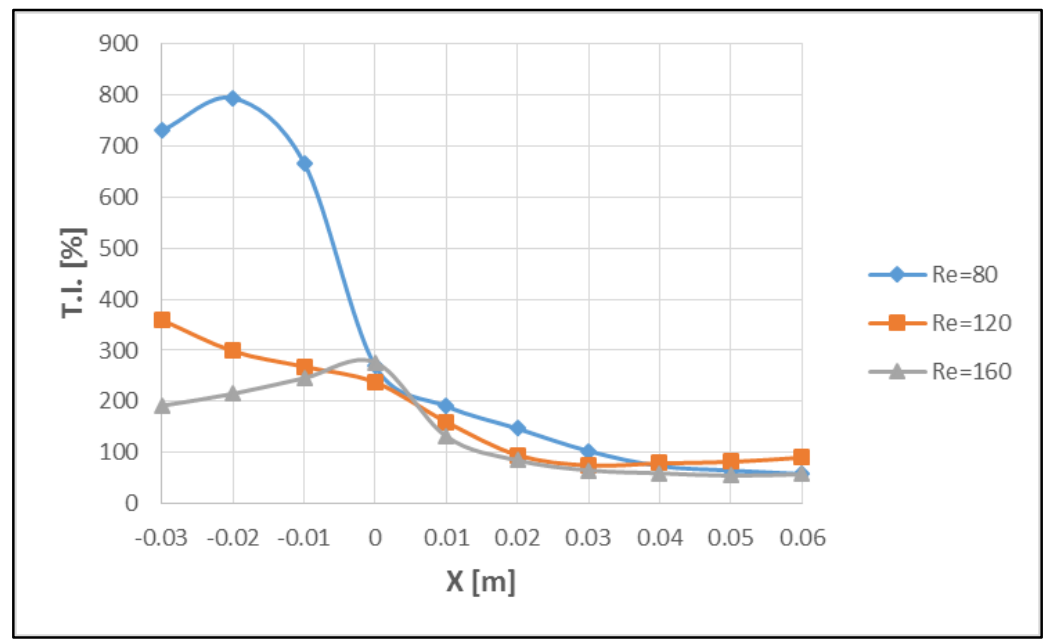

Fig. 11. Turbulence intensity for $\operatorname{Re}=80,120$ and 160 at $\alpha=6$

\subsection{Temperature Distribution}

Fig. 12 shows the temperature distributions for the rotating cylinder at $\alpha=0,2,4$ and 6 when $\mathrm{Re}=80,120$ and 160. The temperature of the cylinder is identical between the different values of Reynolds numbers. Moreover, it can be noted that the variation in surface temperature increases as the rotational rate increases. The experimental results match well with the numerical outcomes.

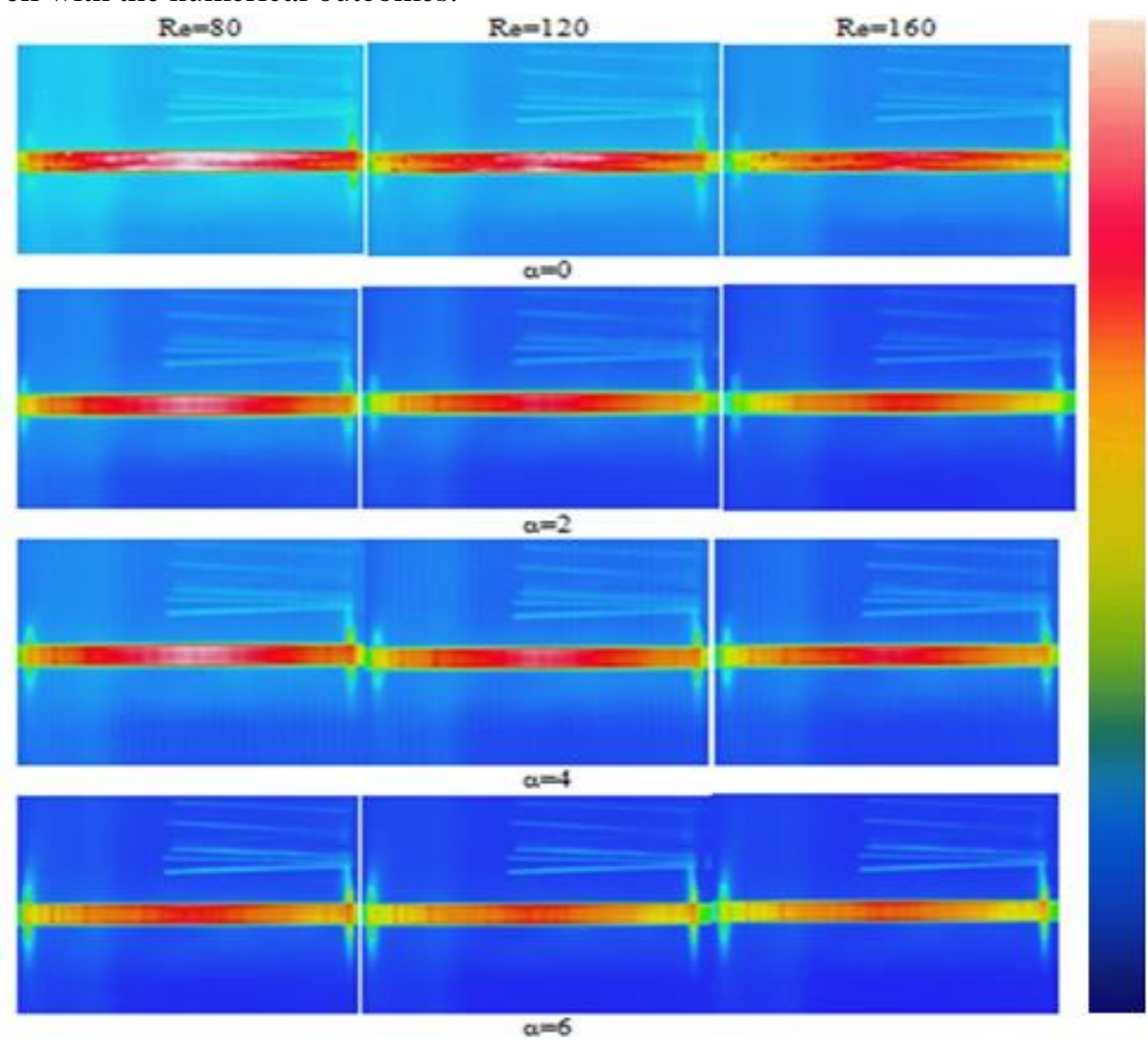

Fig. 12. Thermal camera images at $\mathrm{Re}=80,120$ and 160 and $\alpha=0$. Temperature ranges from $10^{\circ} \mathrm{C}$ to $69^{\circ} \mathrm{C}$. 
Temperature distributions around the cylinder are shown in Fig. 13 a-f. The temperature around the cylinder increase with increasing Reynolds number. Figures also provide a clear image of the effect of the enveloping vortex on temperature distribution aroud the rotating cylinder. The enveloping vortex acts as an insulator for heat transfer between cylinder and free stream.

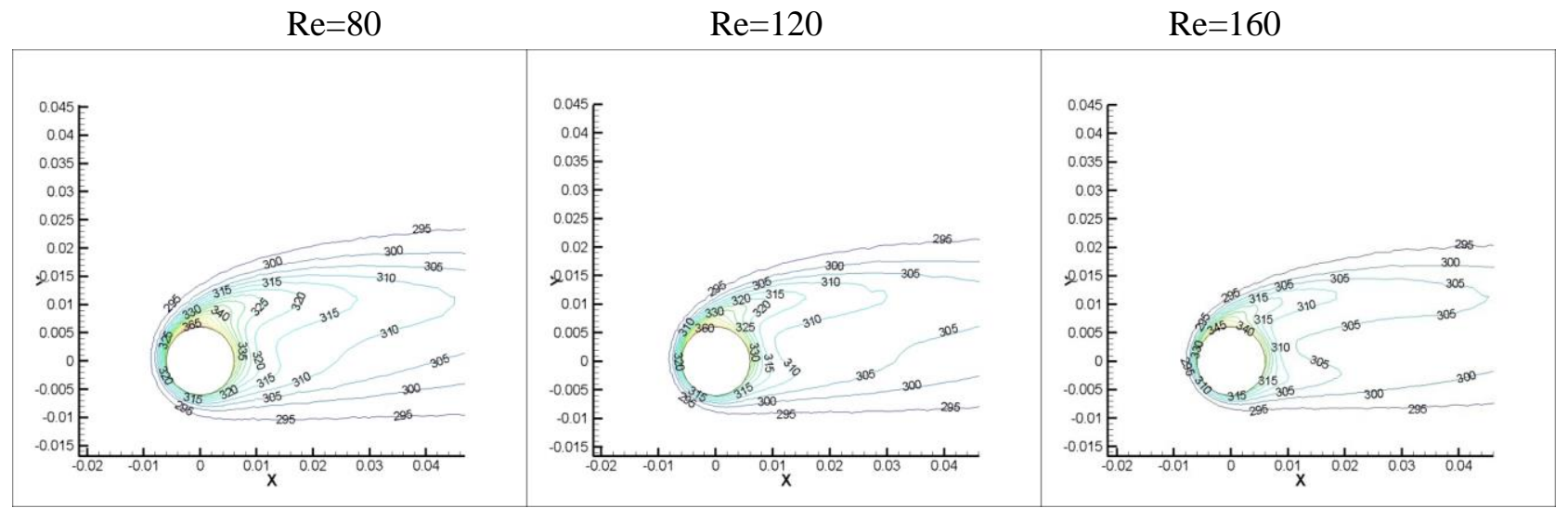

Fig. 13a. Temperature distribution around the cylinder at $\alpha=1$

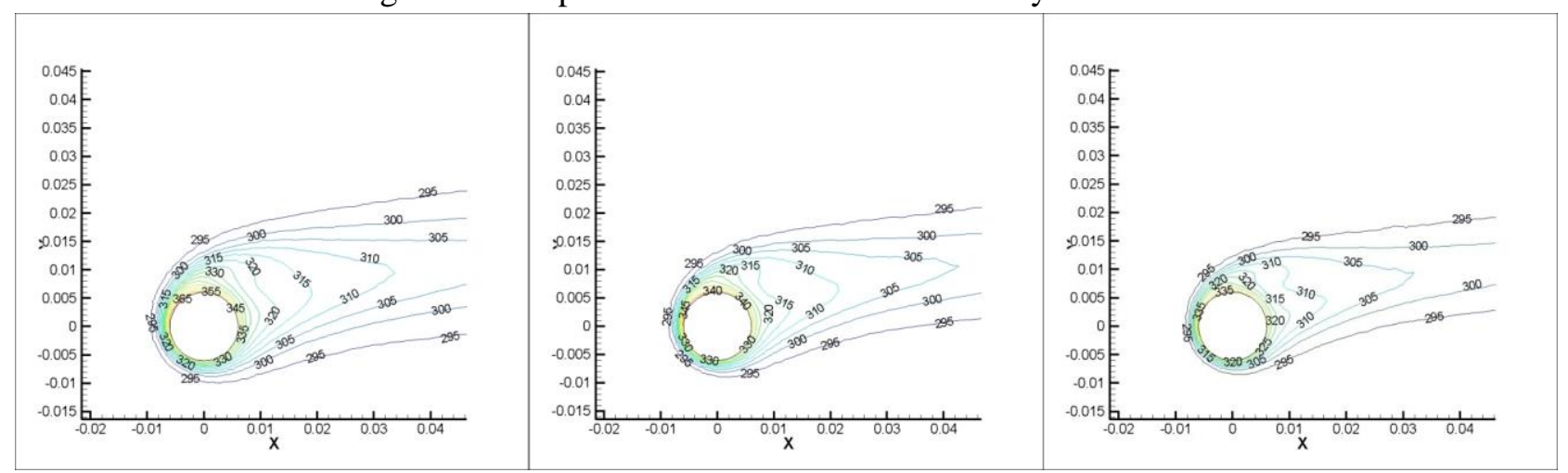

Fig. 13b. Temperature distribution around the cylinder at $\alpha=2$

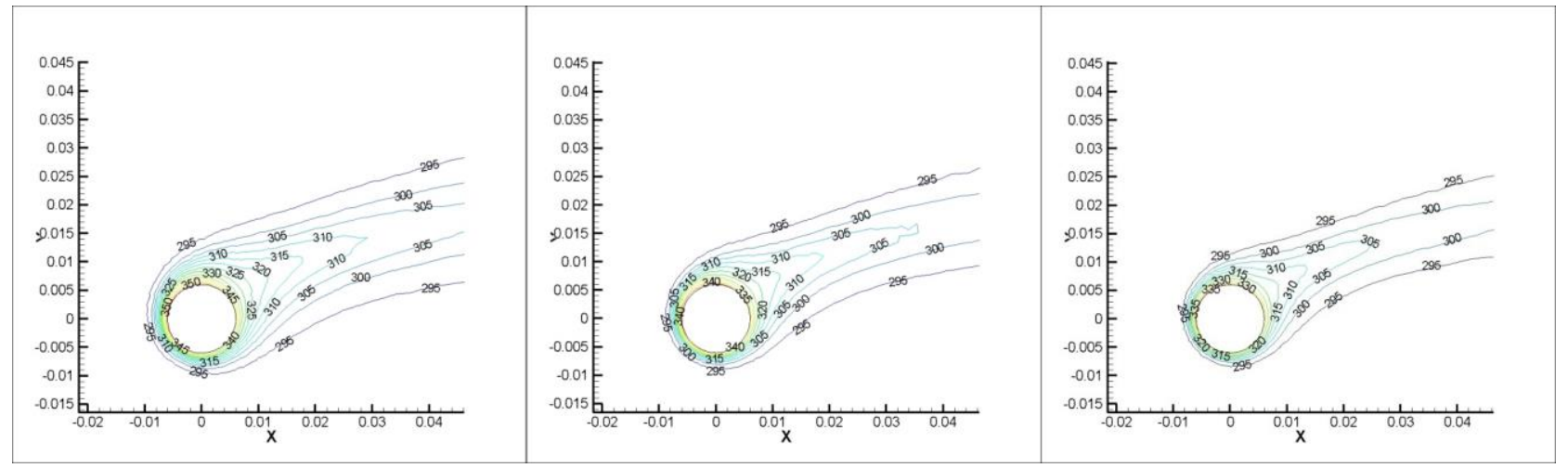

Fig. 13c. Temperature distribution around the cylinder at $\alpha=3$ 

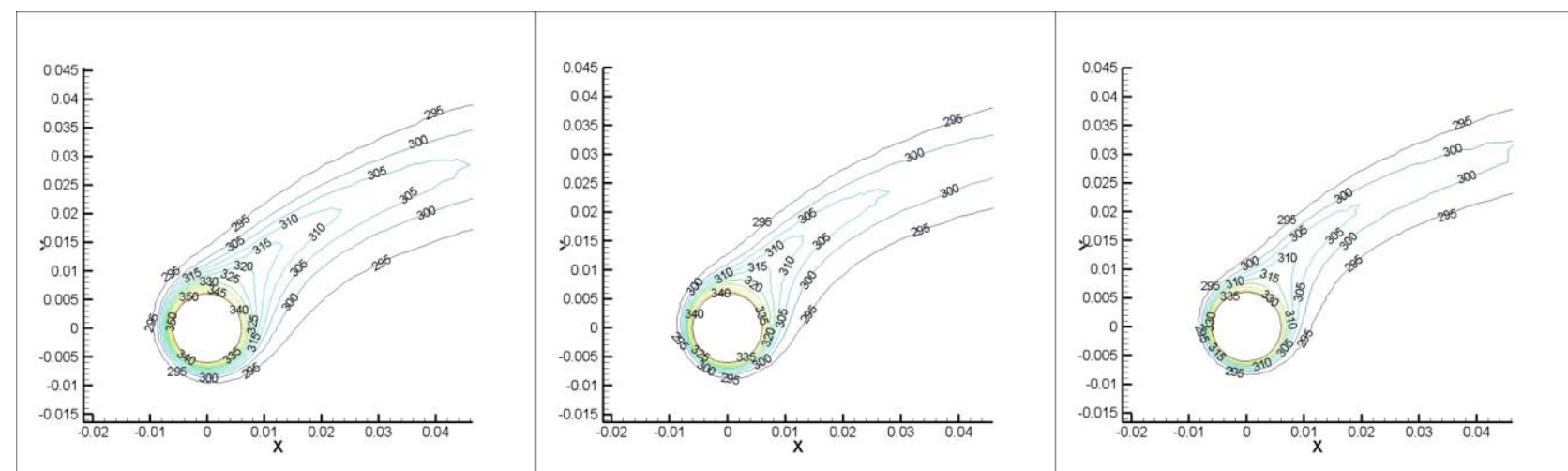

Fig. 13d. Temperature distribution around the cylinder at $\alpha=4$

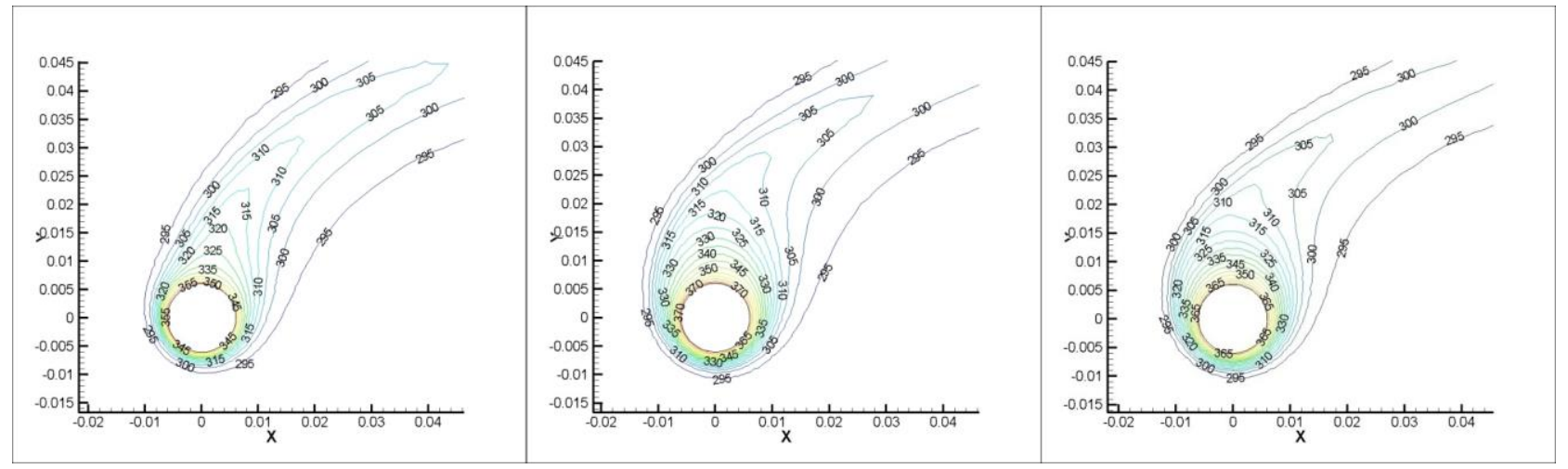

Fig. 13e. Temperature distribution around the cylinder at $\alpha=5$

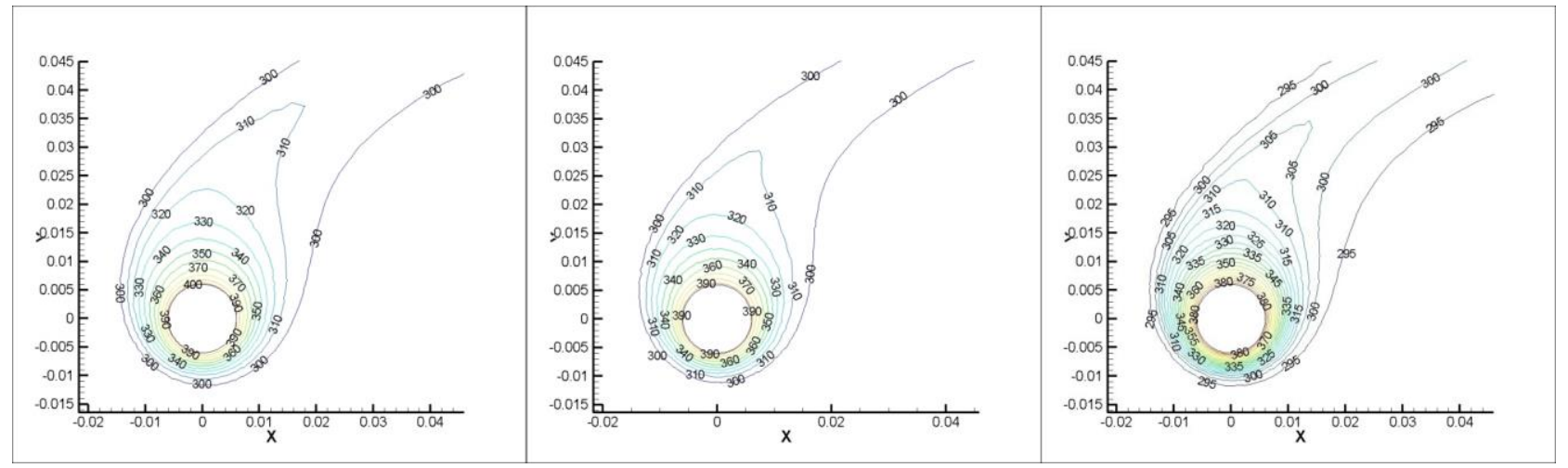

Fig. 13f. Temperature distribution around the cylinder at $\alpha=6$

\subsection{Local Nusselt number}

The measurements necessary for the heat transfer calculations were accomplished, including the electric power to the heater, $q$, the air temperature, $t_{\infty}$, the local temperature obtained from the thermocouples, $t_{n}$, where $\mathrm{n}$ represent the thermocouples location, and the cylinder surface area. The Nusselt number for the uniform heat flux is expressed as: $N u=h D / k$, with the heat transfer coefficient determined as: $h=q / A\left(t_{n}-t_{\infty}\right)$.

The variation of the average Nusselt number is plotted against different $\alpha$ and Re, Fig. 14. In each case the same trend is observed, with the Nusselt number decreasing with an increase of the rotational rate. The figure also shows that the Nusselt increases with increasing Re at a constant $\alpha$ because of the increasing in inertia in the flow. However, at higher rotation rates, i.e. $\alpha=6$, the Nusselt number is almost independent of Reynolds numbers. This is caused by the suppression in vortex shedding and in turn the change in flow structure. Therefore, the thermal resistance and $\mathrm{Nu}$ remain constant. 
In order to provide a clear image to the variation of Nusselt number on the surface of cylinder with increase of the $\mathrm{Re}$ at various rotational rates, polar plots were developed. For the stationary cylinder, $\alpha=0$, Fig. 15 shows a Nusselt number increasing with $\mathrm{Re}$, where $\mathrm{Nu}_{\mathrm{L}}$ is time averaged for $\mathrm{Re}=80,120$ and 160. The variation in the Nusselt number is symmetrical about $\phi=180$ for all $\mathrm{Re}$. Moreover, the maximum $\mathrm{Nu}_{\mathrm{L}}$ was recorded at $\phi=0$ (front stagnation point) for all $\mathrm{Re}$, whereas the minimum value was located at $\phi=180$. For the rotating cylinder the maximum $\mathrm{Nu}_{\mathrm{L}}$ does not occur at the front stagnation point but shifts in the direction of rotation. Further increase in rotation rates shows independence of $\mathrm{Nu}$ with respect to Re, especially at the highest rotation rate $\alpha=6$.

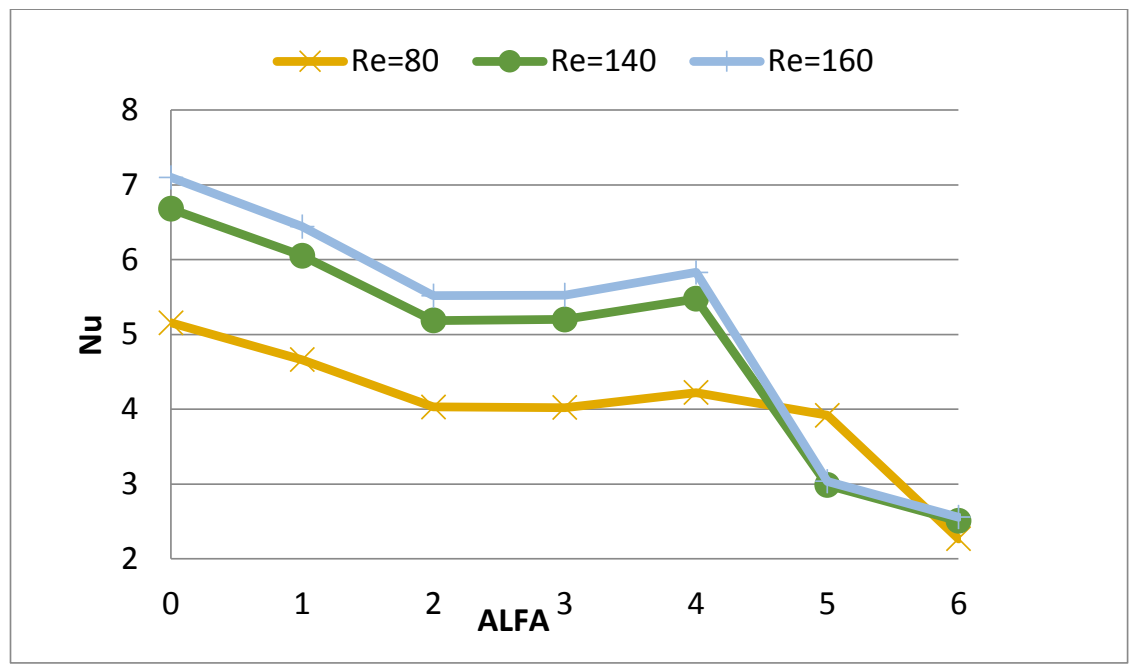

Fig. 14. Local Nusselt number at $\operatorname{Re}=80,120$ and 160

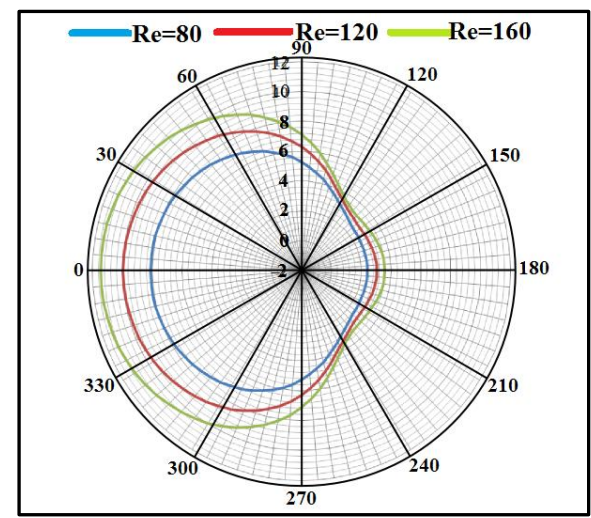

$\alpha=0$

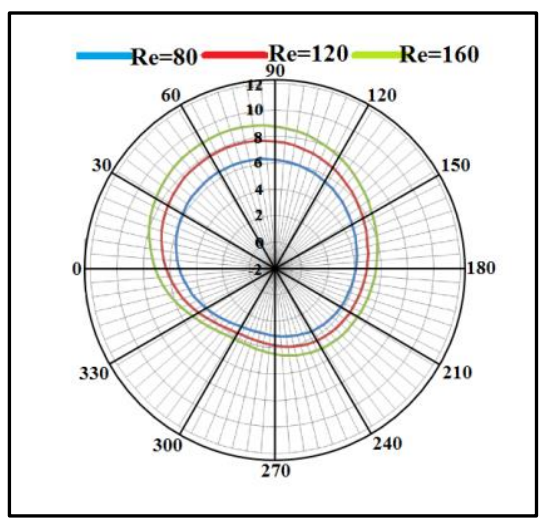

$\alpha=1$

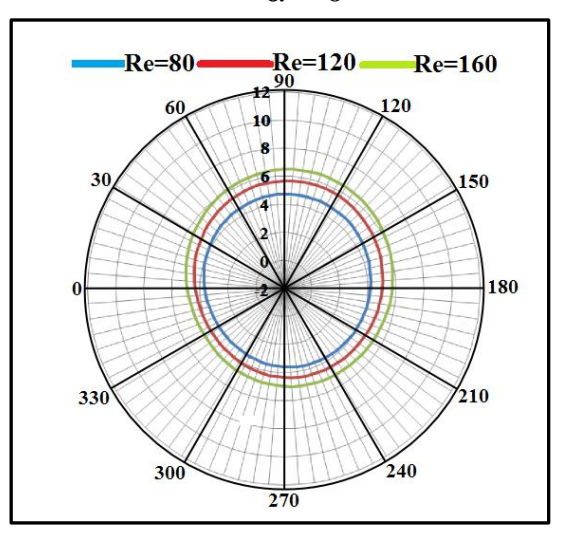

$\alpha=2$

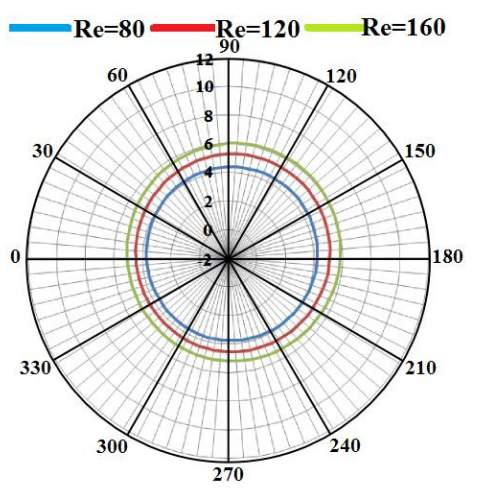

$\alpha=3$ 


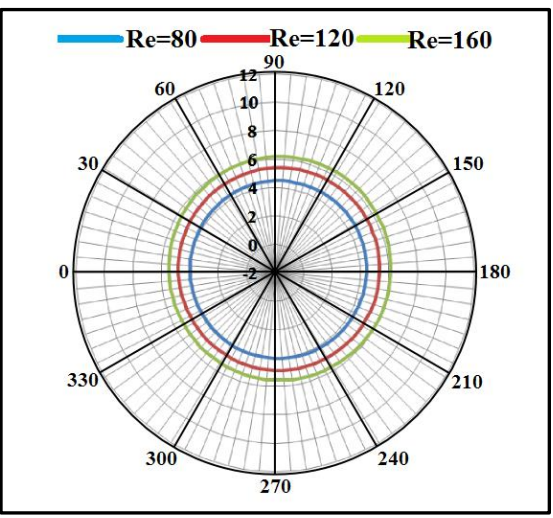

$\alpha=4$

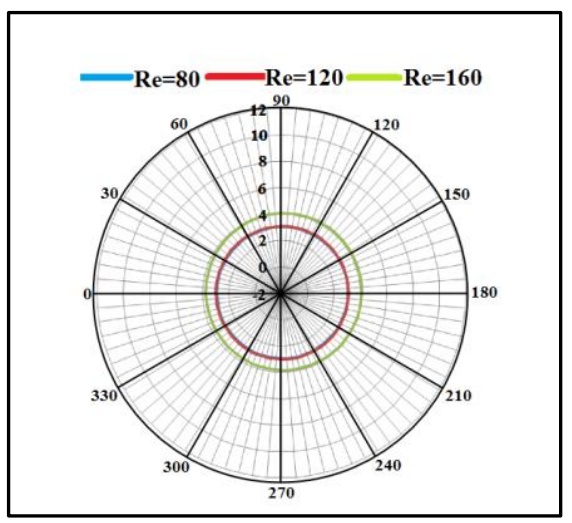

$\alpha=5$

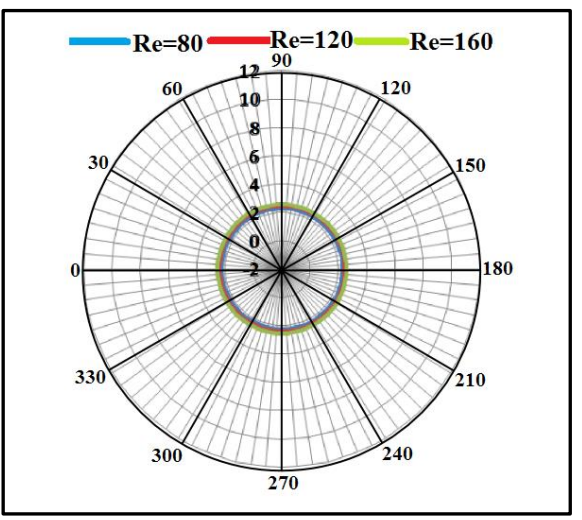

$\alpha=6$

Fig. 15. Polar plot of local Nusselt numbers for various $\alpha$ with increasing Re.

\subsection{Validation of the Present Model}

Extensive comparisons of the present work with other research was carried out, Table 1. The experimental and numerical results of the average $\mathrm{Nu}$ number for stationary and rotating cylinder are in good agreement with the literature review. For stationary cylinders, the maximum discrepancies were in the range of 3.6- to 9.15\%. For the rotating cylinder, the maximum difference between the present results and those of the literature was around 0.26$6.05 \%$. Thus, this validates the present numerical solution procedure, providing confidence in the usage of the model for further applications.

Table 1. Comparison between average Nu obtained in this work and those acquired by Paramane et al. (2012) for

\begin{tabular}{|c|c|c|c|c|c|c|c|c|}
\hline \\
\hline & \multirow{2}{*}{$\mathbf{R e}$} & $\begin{array}{l}\text { Frxea } \\
\text { Cylinder }\end{array}$ & \multicolumn{6}{|c|}{ Rotating Cylinder } \\
\hline & & $\boldsymbol{\alpha}=\mathbf{0}$ & $\alpha=1$ & $\alpha=2$ & $\alpha=3$ & $\alpha=4$ & $\alpha=5$ & $\alpha=6$ \\
\hline $\begin{array}{c}\text { Paramane } \\
\text { and Sharma[12] }\end{array}$ & \multirow[t]{2}{*}{80} & 5.29 & 4.79 & 3.98 & 3.96 & 4.15 & 3.83 & 2.47 \\
\hline Present Work & & 5.1563 & 4.6621 & 4.0299 & 4.0212 & 4.2219 & 3.9195 & 2.2675 \\
\hline $\begin{array}{c}\text { Paramane } \\
\text { and Sharma[12] }\end{array}$ & \multirow[t]{2}{*}{120} & 6.55 & 5.92 & 5.06 & 5.08 & 5.32 & 3.27 & 2.4 \\
\hline Present Work & & 6.444 & 5.6312 & 4.834 & 4.8429 & 5.0984 & 2.9811 & 2.4381 \\
\hline $\begin{array}{c}\text { Paramane } \\
\text { and Sharma[12] }\end{array}$ & \multirow[t]{2}{*}{160} & 7.53 & 6.9 & 5.38 & 5.39 & 5.66 & 3.21 & 2.4 \\
\hline Present Work & & 7.1000 & 6.4428 & 5.5166 & 5.5265 & 5.8289 & 3.0362 & 2.5569 \\
\hline
\end{tabular}

\section{Conclusion}

Flow passing over a circular cylinder, spinning in counter-clockwise, and placed in a uniform flow of $\operatorname{Re}=80$, 120 and 160 has been studied experimentally for various rotation rates $\alpha=0,2,4$ and 6 . The diagnostic was achieved using flow visualization techniques that include High Speed Photography, Thermal Visualization and LDV techniques. It was found that at higher rotational rates turbulence decreases downstream the cylinder, with a high peak just upstream as a consequence of the clashing flows. Moreover, velocity peaks appear at higher Re and $\alpha$, leading to vortical structures that act not only in the formation of zero velocity nodes, but also in the insulation of the rotating cylinder. For stationary cylinder, the average Nusselt number increases as Reynolds number increases. As the rotational speed increases, the average Nusselt number decreases. Results show that for specific Reynolds numbers the maximum average heat rate occurs at the same locations, whilst the minimum value occurs 
at the higher rotational Reynolds number. At higher rotational rates, the Nusselt number is nearly independent of Reynolds number and thermal boundary conditions. Comparison of the results with the previous studies has showed a good agreement between each other, corroborating the model for further studies. Further work could include studies of the effect of higher Reynolds Numbers, rough and dimpled cylinders

\section{Acknowledgements}

The authors gratefully acknowledge the use of instruments from the EPSRC instrument pool

\section{References}

[1] B. V. S. S. S. Prasad, A. A. Tawfek, A. K. Mohanty (1991) Heat transfer form a circular cylinder rotating about an orthogonal axis in quiescent air. Experiments in Fluids, vol. 10, pp 267-272.

[2] Laszlo B. (2003) Computation of unsteady momentum and heat transfer from a fixed circular cylinder in laminar flow. Journal of computational and applied Mechanics, vol. 4, pp 13-25.

[3] C. F. Lange, F. Durst, M. Breuer (1998) Momentum and heat transfer from cylinders in laminar flow cross flow at $10^{-4} \leq \mathrm{Re} \leq 200$. International Journal of Heat Transfer, vol. 41, pp 3409-3430.

[4] Baris O. (2000) Measurements of convective heat transfer coefficient for a horizontal cylinder rotating in quiescent air. International Communications in Heat and Mass Transfer, vol. 27, pp 389-395.

[5] Reda I. E. (2013) Experimental and numerical investigation of heat transfer from a heated horizontal cylinder rotating in still air around its axis. Ain Shams Engineering Journal, vol. 15, pp 177-185.

[6] D. A. Kaminski, X. D. Fu, M. K. Jensen (1995) Numerical and experimental analysis of combined convective and radiative heat transfer in laminar flow over a circular cylinder. International Journal of Heat and Mass Transfer, vol. 38, pp 3161-3169

[7] D. B. Ingham, T. Tang (1990) A numerical investigation into the steady flow past a rotating circular cylinder at low and intermediate Reynolds numbers. Journal of Computational Physics, vol. 87, pp 91-107.

[8] D. Stojkovic, M. Breuer, F. Durst (2002) Effect of high rotation rates on the laminar flow around a circular cylinder. Physics of Fluid, vol. 9, pp 3160-3178.

[9] V. A. F. Costa, A. M. Raimundo (2010) Steady mixed convection in a differentially heated square enclosure with an active rotating circular cylinder. International Journal of Heat and Mass Transfer, vol. 53, pp 12081219.

[10] Chuan-Chieh L., Chao-An L. (2014) Mixed convection of a heated rotating cylinder in a square enclosure. International Journal of Heat and Mass Transfer, vol. 72, pp 9-22

[11] Sachin B. P., Atul S. (2009) Numerical Investigation of heat and fluid flow across a rotating circular cylinder maintained at constant temperature in 2-D laminar flow regime. International Journal of Heat and Mass Transfer, vol. 52, pp 3205-3216.

[12] Sachin B. P., Atul S. (2010) Heat and fluid flow across a rotating cylinder dissipating uniform heat flux in 2-D laminar flow regime. International Journal of Heat and Mass Transfer, vol. 53, pp 4672-4683.

[13] Cheol-Woo P., Sang-Joon L. (2000) Free end effects on the near wake flow structure behind a finite circular cylinder. Journal of Wind Engineering and Industrial Aerodynamic, vol. 88, pp 231-246.

[14] H. M. Badr, S. C. R. Dennis, P. J. S. Young (1989) Steady and unsteady flow past a rotating circular cylinder at low Reynolds numbers. Computers \& Fluids, vol. 17, pp 579-609.

[15] S. Kumar, C. Cantu, B. Gonzalez (2011) Flow past a rotating cylinder at low and high rotation rates. Journal of Fluids Engineering, vol. 133, 041201-1.

[16] Sanjay M, Bhaskar K (2003) Flow past a rotating cylinder. Journal of Fluid Mechanics, vol. 476, pp 303334, DOI 10.1017/S0022112002002938.

[17] Coutanceau M, Menard C (1985) Influence of Rotation on the near wake development behind an impulsively started circular cylinder. Journal of Fluid Mechanics, vol. 158, pp 399-446.

[18] Abdullah A. K. (1996) An approximate solution of the convective heat transfer from an isothermal rotating cylinder. International Journal of Heat and Fluid Flow, vol. 17, pp 439-441. 